\title{
Personality Traits and Self Regulation: A Comparative Study among Current, Relapse and Remitted Drug Abuse Patients
}

\author{
Nadia Bukhtawer, Sumaira Muhammad, Ayesha Iqbal \\ Department of Behavioral Sciences, Fatima Jinnah Women University, Rawalpindi, Pakistan \\ Email: nadia azhar@hotmail.com
}

Received 28 April 2014; revised 30 May 2014; accepted 6 June 2014

Copyright (C) 2014 by authors and Scientific Research Publishing Inc.

This work is licensed under the Creative Commons Attribution International License (CC BY).

http://creativecommons.org/licenses/by/4.0/

(c) () Open Access

\section{Abstract}

Objective: The current study aimed to investigate the relationship among personality traits and self regulation among different drug abuse cases. The three categories of drug abuse were based on phases of addiction namely current, relapse and remitted cases. Method: The sample consisted of 108 drug abusers taken from different rehabilitation centers of Rawalpindi and Islamabad. The selected drug abusers were at current $(n=40)$, relapsed $(n=42)$ and remission phase $(n=27)$ of drug addiction; with an age range of 20 to 70 years. The data were collected from private rehabilitation centers of Rawalpindi and Islamabad. In this study, Eysenck Personality Questionnaire Revised EPQR-Short (Eysenck, Eysenck \& Barrett, 1985) was used to measure personality traits of the drug abuse cases. Self Regulation Questionnaire (SRQ) developed by Miller and Brown (1991) was used to measure the self regulatory skills. The data were collected from rehabilitation centers. Before collection of data, formal permission was taken from the administration of these centers. Research protocol included an Ethical Pro-forma explaining rationale of study, ensuring confidentiality of the information to be used for research purpose only and also had personal consent form. In the first phase, pilot study was conducted to check the reliability of translated scale and availability of sample. A sample of $\mathbf{1 5}$ drug abusers was selected for pilot study. In the next step (main study); protocol was administered on current, relapsed and remitted cases through individual administration. For remitted cases, the researcher consulted authorities (administration head, and medical doctors) of centers and administered protocol on the days when these participants came for follow-up session. Results: The results of the study indicate strong correlation between self regulation and personality traits $\left(r=0.27^{* *}\right)$. Furthermore, most of the participants scored higher on Psychoticism (100\%), Introversion (75\%) while Neuroticism (58\%) trait was found less among drug abuse cases. The study also indicates strong correlation between self regulation and Extraversion-Introversion personality trait $\left(r=0.20^{* *}\right)$ while other traits did not have significant correlations with self regulation. Furthermore, the descriptive frequencies reveal that most of the participants scored higher on Psychoticism (100\%), Introversion (75\%) while Neuroticism (58\%) 
trait was found less among drug abuse cases. Conclusion: From the above mentioned findings, it is concluded that self regulation is associated with personality traits among drug abusers. The comparisons of personality traits and self regulatory skills among the different phases of addiction provide an insight into how these traits interact with self control to regulate impulsive and addictive behaviors.

\title{
Keywords
}

\author{
Addiction, Personality Traits, Self Regulation, Drug Abuse
}

\section{Introduction}

In Pakistan, the growing problem of drug abuse in general [1] and specifically rising numbers of abusers in youth [2] has led to a lot of research in this area to understand this complex phenomenon. One such emerging area of interest has been drug abuse and its association with personality traits [3]. A growing body of research has established that specific personality traits such as impulsivity, neuroticism and extraversion are found among individuals with drug addiction [4]-[9]. However, understanding the dynamics of personality with drug abuse is complex and traits may vary in different conditions such as choice of drugs, frequency and intensity [10]. Exploring personality traits further in drug addicts, one common factor was found to be linked to them; the ability to regulate oneself is known to be associated with specific personality traits [11].

Self regulation is considered a regulating factor in development of specific personality traits [11] [12]. It also increases the probability of drug abuse in self regulation which is known to regulate self control [13] as well regulate the autonomy and impulse-control capacity [14]. Inadequate self regulatory skills have been associated with drinking, smoking, drug abuse and health risk behaviors and drug abusers have been found to have relatively low self control as compared to general population [15] [16].

Since personality traits and self regulation both have a strong association with drug abuse, it becomes essential to understand the dynamics of these two factors and how they both contribute to development and regulation of drug abuse. However, these variables have not been studied extensively in this regard and created a knowledge gap. The current study undertakes the aim to explore the association of self regulation and personality traits in individuals with drug abuse. Since, low and high levels of regulation affect the use and consumption of drugs as well as the ability to practice self control [17], it is important to study these among current, remitted and relapse cases of drug abuse [18].

In Pakistani context, a lot of focus has been on describing and exploring addiction with reference to psychological disorders [19]-[21] but understanding its complex association with personality traits and comparisons of self regulatory skills among current, relapse and remitted users is yet to be explored. The current study aims to explore and compare personality traits and its association with self regulation among current, remitted and relapse cases.

\section{Method}

The present research is descriptive and co relational examining the association of different personality traits of drug abusers with their self regulation.

\subsection{Participants}

Sample consisted of 108 adult drug abusers taken from different rehabilitation centers of Rawalpindi and Islamabad. The selected rehabilitation centers are private offering inpatient and outpatient (residential) services; including detoxification and psychosocial intervention. The selected drug abusers were at current $(\mathrm{n}=40)$, relapsed $(n=42)$ and remission phase $(n=27)$ of drug abuse. Current and relapse cases were admitted in rehabilitation centers whereas remitted participants were coming for follow-ups in these centers. Current and relapse cases included in the study were not in initial phase of detoxification. All participants were male as the number of females in rehabilitation centers was scarce; because of social issues of stigmatization and taboo. 


\subsection{Instruments}

\section{Demographic Form}

The demographic form was developed in order to obtain basic information about the participants. It consisted of demographic variables including age, marital status, occupation, education, and phase of abuse (current, relapse and remitted cases).

\subsection{Eysenck Personality Questionnaire Revised (EPQR)}

EPQR-Short [22] is a self reported personality questionnaire. It has 48 items, measuring personality traits. It consisted of four subscales; E (Extraversion vs. Introversion), N (Neuroticism or "Emotionality") P (Psychoticism or "Tough Mindedness)", L (Lie scale). Each subscale has 12 items. L scale was used to measure the tendency on part of the participants to "fake good" or respond in a socially desirable manner. All items had a binary response, "yes" or "no". The alpha reliability of Eysenck's personality questionnaire—revised was 0.70 [22]. The cutoff for Extroversion, Neuroticism and Psychoticism is 5 and for the present study; Urdu translated version done by Kausar was used [23].

\subsection{Description of Subscales}

Participants scoring high on extraversion subscale are found to be social, outgoing, impulsive, risk takers while low scorers are introverts; they are quiet, retiring and studious. High scores on neuroticism indicate emotional instability; having worries and anxieties whereas low scores indicate emotional stability. Scoring high on Psychoticism subscale indicates ego depletion; such individuals are considered cruel, socially indifferent, hostile and aggressive. While those having low scores on this subscale have strong ego control [22].

\subsection{Self Regulation Questionnaire (SRQ)}

Self Regulation Questionnaire SRQ was developed by Miller \& Brown [24] based on seven-step model of self regulation; which are receiving information, evaluation of relevant information, triggering change, searching for options, formulating a plan, implementing a plan, assessing the plan effectiveness, repeating 1 \& 2 . It consists of 63 items scored on a 5-point Likert scale. The recommended ranges for interpreting SRQ total scores are as follows; $\geq 239$ = High (intact) self regulation capacity, 214 - 238 = Intermediate (moderate) self regulation capacity, $\leq 213$ = Low (impaired) self regulation capacity. The scale has sound psychometric properties; the alpha reliability of Self Regulation Questionnaire found to be 0.91 and has strong content validity [25].

\subsection{Scale Translation}

For the current study scale was translated into Urdu through the process of back translation. In first step, forward translation was carried out; scale was translated into Urdu independently by two bilinguals. From these two translations, best translation was selected with consensus of researchers and two other psychologists from the department of behavior science, Fatima Jinnah Women University. In next step, the selected Urdu version was given to two other bilinguals for backward translation. The purpose of backward translation was to determine whether the translated version was valid and the items matched with the original version or not. The backward translated versions were than compared with original version; the translation matched with the original except from one item; that was modified and simplified. The translated version was administered on 15 individuals to find out its reliability of the scale. The reliability came out to be 0.71 .

\subsection{Procedure}

After the approval by University Ethical Board, the data was collected from rehabilitation centers. Before collection of data, formal permission was taken from the administration of these centers. Research protocol included an informed consent form explaining rationale of study, ensuring confidentiality of the information to be used for research purpose only and also had personal consent form. In the first phase, pilot study was conducted to check the reliability of translated scale and availability of sample. A sample of 15 drug abusers was selected for pilot study. In the next step (main study); protocol was administered on current, relapsed and remitted cases through individual administration. For remitted cases, the researcher consulted authorities (administration head, 
and medical doctors) of centers and administered protocol on the days when these participants came for follow-up session. The average time for administration of protocol was 15 - 25 minutes and data was collected in 30 days.

\section{Results}

Descriptive analysis of the data (See Table 1) revealed that, majority of drug abusers (60\%) current, $64 \%$ relapsed and $81 \%$ of remitted cases were young. Most of the current drug abusers were unmarried (60\%), having bachelor's degree (32\%) and were professionals (77\%), whereas most of the Relapse cases were married (48\%) educated under matriculation (38\%) and were skilled labors (74\%). Among remitted cases, 69\% were unmarried having education up to matriculation (61\%) and were skilled labors. The analysis also depicted that, $75 \%$ of the current, $69 \%$ of relapsed and $61 \%$ of remitted participants were introverts (See Table 2). Among the participants, more percentage of participants was emotionally stable among relapse (69\%) and remitted (61\%) cases. All current, remitted and relapsed participants had low ego control.

The level of self regulation among current, relapse and remitted cases are presented in Table 3 . The chi square analysis depicted significant differences $(\mathrm{p}=0.16)$ of self regulation among current, relapsed and remitted cases; current users (55\%) had moderate self regulation whereas relapsed (64\%) and remitted cases (65\%) had impaired self regulation.

Correlation between self regulation scores (SRQ) and personality traits scores of Revised Eysenck personality questionnaire and EPQR subscales is reported in Table 4. The correlation between total of SRQ and total of EPQR is significant $\left[r(108)=0.27^{* *}, p<0.01\right]$. Similarly, highly significant inverse correlation $[r(108)=-0.001, p$ $<0.001$ ] between self regulation (SRQ) and Psychoticism (TPSY) subscale was found. Self regulation also correlated positively with extraversion (TEXT) subscale $\left[r(108)=0.20^{*}, \mathrm{p}<0.05\right]$. However, non significant inverse correlation $[r(108)=-0.08, p<0.05]$ between self regulation and Neuroticism subscale (TNEU) were found.

\section{Discussion}

The current study aimed to investigate the relationship among personality traits and self regulation among different drug abuse cases. The three categories of drug abuse were based on phases of addiction namely current, relapse and remitted cases. Since, the main aim was to explore how self regulation and personality traits regulate the use and abuse of drugs, thus comparisons among the current, relapse and recovering users of drugs gives a more comprehensive understanding of the role of personality traits and self regulation among drug abuse patients.

Table 1. Frequencies and percentages of demographic characteristics of participants $(\mathrm{N}=108)$.

\begin{tabular}{cccc}
\hline Characteristics & Current $(\mathrm{N}=40) \mathrm{f}(\%)$ & Relapse $(\mathrm{N}=42) \mathrm{f}(\%)$ & Remitted $(\mathrm{N}=26) \mathrm{f}(\%)$ \\
\hline Age & & & $21(81)$ \\
$21-30$ & $22(55)$ & $27(64)$ & $5(19)$ \\
$31-40$ & $13(32)$ & $9(21)$ & $0(00)$ \\
$41-50$ & $4(10)$ & $4(9)$ & $0(00)$ \\
51 and above & $1(2)$ & $2(5)$ & $4(15)$ \\
& & & $18(69)$ \\
Marital Status & $13(32)$ & $20(48)$ & $4(15)$ \\
Married & $24(60)$ & $16(38)$ & $1(4)$ \\
Unmarried & $3(7)$ & $6(14)$ & $10(38)$ \\
Divorced/Separated & & $13(50)$ \\
Education & $12(30)$ & $15(36)$ & $2(8)$ \\
Under matriculation & $7(17)$ & $8(19)$ & $16(61)$ \\
Matriculation & $8(20)$ & $8(19)$ & $2(8)$ \\
Intermediate & $13(32)$ & $31(74)$ & $8(31)$ \\
Bachelors & $20(50)$ & $7(17$ & $4(15)$ \\
Employment status & $31(77)$ & & \\
Skilled labor & $8(20)$ & & \\
Professional & & & \\
Student & & &
\end{tabular}


Table 2. Chi square and cross tabulation on personality traits among current, remitted and relapse cases $(\mathrm{N}=108)$.

\begin{tabular}{cccccc}
\hline Personality Traits & $\begin{array}{c}\text { Current } \\
\mathrm{f}(\%)\end{array}$ & $\begin{array}{c}\text { Relapse } \\
\mathrm{f}(\%)\end{array}$ & $\begin{array}{c}\text { Remitted } \\
\mathrm{f}(\%)\end{array}$ & $\mathrm{X} 2$ & $\mathrm{p}$ \\
\hline Extroverts & $10(25)$ & $13(31)$ & $10(38)$ & & \\
Introverts & $30(75)$ & $29(69)$ & $16(61)$ & 1.4 & 0.6 \\
Stable & $18(45)$ & $29(69)$ & $16(61)$ & & \\
Unstable & $22(55)$ & $13(31)$ & $10(38)$ & 5.0 & 0.08 \\
Low ego control & $40(100)$ & $42(100)$ & $26(100)$ & -- & - \\
\hline
\end{tabular}

$\mathrm{df}=2$.

Table 3. Chi square and cross tabulation on self regulation based on drug abuse $(\mathrm{N}=108)$.

\begin{tabular}{cccccc}
\hline Personality Trait & Current $\mathrm{f}(\%)$ & Relapse $\mathrm{f}(\%)$ & Remitted f(\%) & $\mathrm{X} 2$ & $\mathrm{p}$ \\
\hline Extroverts & $10(25)$ & $13(31)$ & $10(38)$ & & \\
Introverts & $30(75)$ & $29(69)$ & $16(61)$ & 1.4 & 0.6 \\
Stable & $18(45)$ & $29(69)$ & $16(61)$ & & \\
Unstable & $22(55)$ & $13(31)$ & $10(38)$ & 5.0 & 0.08 \\
Low ego control & $40(100)$ & $42(100)$ & $26(100)$ & -- & -- \\
\hline
\end{tabular}

$\mathrm{df}=2$.

Table 4. Pearson product correlations between total of SRQ, total of EPQR and subscales of EPQR $(\mathrm{N}=108)$.

\begin{tabular}{ccccccc}
\hline Sr No. & Scales/Subscales & 1 & 2 & 3 & 4 & -0.08 \\
1 & TRSQ & - & $0.27^{* *}$ & $0.20^{*}$ & -0.001 \\
2 & TEPQR & - & - & $-0.54^{*}$ & $-0.55^{* *}$ & $0.31^{* *}$ \\
3 & TEXT & - & - & - & -10 & -0.16 \\
4 & TNEU & - & - & - & - \\
5 & TPSY & - & - & - & - \\
\hline
\end{tabular}

${ }^{*} \mathrm{p}<0.05,{ }^{* *} \mathrm{p}<0.01$. Note: TRSQ = Total of Self Regulation Questionnaire, TEPQR $=$ Total of Revised Eysenck Personality Questionnaire, TEXT $=$ Total of Extraversion, TNEU $=$ Total of Neuroticism \& TPSY $=$ Total of Psychoticism.

The results of the study reveal that most of the current users were in the younger age group (20 - 30 yrs). These findings are consistent with prior studies indicating that drug abuse is more common among youth in Pakistan especially early adolescence and young adulthood [26] [27]. A number of factors identified for high drug abuse among Pakistani youth include parent's indulgence in drugs, psychosocial distress, disturbed family relations and conflict, high drug abuse among peers, high sensation seeking behaviors and problems in adjustment [28] [29]. This also exacerbated by easy illegal availability of drugs in Pakistan; due to high opium production and trafficking from Afghanistan [30]. Majority of the cases were also unmarried which is consistent with previous studies [27] [31]. Drug abuse can stigmatize their marital prospects and difficulty in finding a life partner can be one reason [27]. In Pakistani culture, man is the bread earner of the family and since most of drug abuse cases have disrupted lifestyle, they cannot afford to begin a family life [32].

Our main hypothesis that personality traits will have a strong correlation (see Table 3) with self regulation. Zimmerman [33] suggested an association among personality traits and the capacity of self regulation. Some have even suggested a theoretical assumption of how self regulation contributes to the development of specific personality traits and temperaments [34]. The present study also builds support for this theoretical assumption as the ability to regulate oneself impacts traits such as impulsivity, stability and ego control as well. Many researchers have found associations among low self regulatory skills and drinking, smoking and drug abuse [17]. The frequencies (See Table 1) of different traits prevalent in current, relapse and remitted drug abuse cases suggest that higher percentage lies among introverts, emotionally unstable and low ego control as well as impaired self regulation.

The only strong correlation between self regulation and EPQR subscale was found on Extraversion-Introversion personality trait $\left(r=0.20^{* *}\right)$. Although self regulation did not significantly correlate with other subscales of Eysenck personality Questionnaire Revised, however, the frequencies of (See Table 1) of different categories of drug abuse give an idea about the dispersion of various personality traits. Psychoticism was found to be 
higher among all categories of drug abuse patients while Extraversion to be lower among drug abuse cases. Evidence from other studies indicates similar patterns. Madhuri [35] found addicts to be more neurotic as well as less impulsive scoring high on Psychoticism and Neuroticism subscales while they are more introvert as compared to non addicts. Hulburt [36] found Psychoticism to be higher among alcoholics while extraversion was lower. Similarly, Speilberger [37] found Psychoticism trait to be more prevalent in smokers as well. Baumeister [14] explained this phenomenon and proposed that ego depletion can lead to less control over one's impulsive and appetitive behaviors which is prevalent among addiction cases.

In the current study, all drug abuse categories had lower score on Neuroticism (See Table 1) which is contrary to previous literature. Instability is usually higher among drug abuse cases [36] [38]. High scores on neuroticism indicate emotional stability and two possible explanations for these findings arise. One, since our sample consisted of majority of relapse and remitted cases, their scores are lower on neuroticism and only current users scored higher in this subscale (See Table 1). Secondly, individuals in relapse and remission cases, being in therapy have gone through detoxification as well as under therapeutic interventions and living in a controlled environment; they may report being in a more stable state which can contribute to higher scores on neuroticism. Also, cultural variations and social desirability can also contribute to these findings.

\section{Conclusions}

The current study provides a general picture of the personality traits of drug abusers. It further provides an insight into the role of self regulation skills in the development, maintenance and abstinence of drug addiction. The comparison of self regulation and personality traits among the drug abusers in three phases of addiction opens up a new topic of research to be explored. Future studies need to explore in depth how self regulation can mediate the development of specific personality traits such as impulsivity, neuroticism which are usually found more among addicts. This has implications for prevention and rehabilitation strategies for addiction.

Although the sample size was limited, still the current study has contributed to the gap of knowledge in Pakistan regarding addiction. Future studies on larger samples can be conducted to explore the phenomenon of addiction with respect to demographics variables as well.

\section{References}

[1] UNODC-ANF (2007) PROBLEM DRUG USE IN PAKISTAN: Results from Year 2006 National Assessment. Anti Narcotics Force in collaboration with UNODC, Islamabad, 1,7,9.

[2] Akhter, A. (2013) Relationship between Drug Use and Self-Esteem. International Journal of Scientific \& Engineering Research, 4.

[3] Conway, K.P., Swendsen, J.D., Rounsaville, B.J. and Merikangas, K.R. (2002) Personality, Drug of Choice and Comorbid Psychopathology among Substance Abusers. Drug \& Alcohol Dependence, 65, 225-234. http://dx.doi.org/10.1016/S0376-8716(01)00168-5

[4] Graham, T. and Strenger, V. (1998) MMPI Characteristics of Alcoholics: A Review. Journal of Consulting and Clinical Psychology, 56, 197-205. http://dx.doi.org/10.1037/0022-006X.56.2.197

[5] Martin, E. and Sher, K.J. (1994) Family History of Alcoholism, Alcohol Use Disorders, and the Five Factor Model of Personality. Journal of Studies on Alcohol, 55, 81-90.

[6] Scourfield, J., Stevens, D.E. and Merikangas, K.R. (1996) Substance Abuse, Comorbidity, and Sensation Seeking: Gender Differences. Comprehensive Psychiatry, 37, 384-392.

[7] McGue, M. (1999) The Behavioral Genetics of Alcoholism. Current Directions in Psychological Science, 8, $109-115$. http://dx.doi.org/10.1111/1467-8721.00026

[8] Fisher, P.A., Stoolmiller, M., Gunnar, M.R. and Burraston, B.O. (2007) Effects of a Therapeutic Intervention for Foster Preschoolers on Diurnal Cortisol Activity. Psychoneuroendocrinology, 32, 892-905. http://dx.doi.org/10.1016/j.psyneuen.2007.06.008

[9] Liraud, F. and Verdoux, H. (2000) Which Temperamental Characteristics Are Associated with Substance Use in Subjects with Psychotic and Mood Disorders? Psychiatry Research, 93, 63-72. http://dx.doi.org/10.1016/S0165-1781(99)00120-1

[10] Mirin, S.M., Weiss, R.D., Griffin, M.L. and Michael, J.L. (1991) Psychopathology in Drug Abusers and Their Families.Comprehensive Psychiatry, 32, 36-51. http://dx.doi.org/10.1016/0010-440X(91)90068-N

[11] Denissen, J.J.A., Penke, L. and Van Aken, M.A.G. (2013) Self-Regulation Underlies Temperament and Personality: 
An Integrative Developmental Framework. Child Development Perspectives, 7, 255-260. http://dx.doi.org/10.1111/cdep.12050

[12] Posner, M.I. and Rothbart, M.K. (2007) Educating the Human Brain. APA Books, Washington DC. http://dx.doi.org/10.1037/11519-000

[13] Baumeister, R.F., Heatherton, T.F. and Tice, D.M. (1994) Losing Control How and Why People Fail at Self-Regulation. Academic Press, San Diego.

[14] Penley, J.A., Tomaka, J. and Wiebe, J.S. (2002) The Association of Coping to Physical and Psychological Health Outcomes: A Meta-Analytic Review. Journal of Behavioral Medicine, 25, 551-603. http://dx.doi.org/10.1023/A:1020641400589

[15] O'leary, A., Goodhart, F., Jemmott, L.S. and Boccher-Lattimore, D. (1992) Predictors of Safer Sex on the College Campus: A Social Cognitive Theory Analysis. Journal of American College Health, 40, 254-263. http://dx.doi.org/10.1080/07448481.1992.9936290

[16] Baumeister, R.F., Vohs, K.D. and Tice, D.M. (2007) The Strength Model of Self-Control. Current Directions in Psychological Science, 16, 351-355.

[17] Hustad, J., Carey, K., Carey, M. and Maisto, S. (2009) Self-Regulation, Alcohol Consumption, and Consequences in Collegestudent Heavy Drinkers: A Simultaneous Latent Growth Analysis. Journal of Studies on Alcohol and Drugs, 70, 373-382.

[18] Hopwood, C.J., Morey, L.C., Skodol, A.E., Stout, R.L., Yen, S., Ansell, E.B., et al. (2007) Five-Factor Model Personality Traits Associated with Alcohol-Related Diagnoses in a Clinical Sample. Journal of Studies on Alcohol and Drugs, 68, 455-460.

[19] Asghar, S., Taj, R., Akhter, S. and Khan, A. (2005) Can Cannabis Abuse Lead to Anxiety and Depression? Journal of Pakistan Psychiatric Society, 2.

[20] Alam, A.Y., Iqbal, A., Mohamud, K.B., Laporte, R.E., Ahmed, A. and Nishtar, S. (2008) Investigating Socio-Economic-Demographic Determinants of Tobacco Use in Rawalpindi, Pakistan. BMC Public Health, 8, 50. http://dx.doi.org/10.1186/1471-2458-8-50

[21] Niazi, R.S., Pervaiz, R., Minhas, F.A. and Najam, N. (2005) Locus of Control and Personality Traits of Male Substance Abusers and Non Abusers. Journal of Pakistan Psychiatric Society, 2, 41.

[22] Eyesenck, H.J. and Eysenck, M.W. (1985) Personality and Individual Differences. A Natural Science Approach. Alcoholism: Clinical \& Experimental Research, 18, 429. http://dx.doi.org/10.1007/978-1-4613-2413-3

[23] Kousar, R. (2001) Eysenck Personality Questionnaire (Urdu Translation and Adaptation in Pakistan). Unpublished Questionnaire, University of the Punjab, Punjab.

[24] Miller, W.R. and Brown, J.M. (1991) Self-Regulation as a Conceptual Basis for the Prevention and Treatment of Addictive Behaviours. In: Heather, N., Miller, W.R. and Greeley, J., Eds., Self-Control and the Addictive Behaviours, Maxwell Macmillan Publishing Australia, Sydney, 3-79.

[25] Carey, K.B., Neal, D.J. and Collins, S.E. (2004) A Psychometric Analysis of the Self-Regulation Questionnaire. Addictive Behaviors, 29, 253-260.

[26] Bashir, A.M. (2005) Socio-Cultural and Psychological Factors of Drugs Abusers; A Case Study of Nijat Markaz Rawalpindi. Unpublished M.Sc. Thesis, University of Arid Agriculture, Rawalpindi.

[27] Salik, M.H., Maan, A.A., Akram, M.B., Muhammad, R.M.F. and Ali, M. (2004) Determinants and Consequences of Drug Addiction in Faisalabad-Pakistan. International Journal of Agriculture and Biology, 6.

[28] Gillis, J.S. and Mubashar, M.H. (1995) Risk Factors of Drug Factors in Pakistan: A Replication. Psychological Reports, 76, 98-108. http://dx.doi.org/10.2466/pr0.1995.76.1.99

[29] Niaz, U., Siddiqui, S.S., Hassan, S., Hussain, H., Ahmed, S. and Akhter, R. (2005) A Survey of Psychosocial Correlates of Drug Abuse in Young Adults Aged 16-21, in Karachi: Identifying "High Risk” Population to Target Intervention Strategies. Pakistan Journal of Medical Sciences, 21, 271-277.

[30] Mubeen, C. and Sharif, M. (2007) Drug Addiction among Females in District Lahore (Pakistan). Journal of Agriculture and Social Sciences, 3, 101-103.

[31] Habib, F. (1998) Psychological and Social Aspects of Drug Addiction. Reflection on Drug Abuse, N.N.C.B., Islamabad.

[32] Akhtar, S., Kashif, A., Arif, A. and Khan, A.F. (2012) Impact of Long Working Hours on Family Wellbeing of Corporate Family. World Applied Sciences Journal, 16, 1302-1307.

[33] Zimmerman, B.J. (2000) Attainment of Self-Regulation: A Social Cognitive Perspective. In: Boekaerts, M., Pintrich, P.R. and Zeidner, M., Eds., Handbook of Self-Regulation, San Diego, Academic Press, 13-39. 
http://dx.doi.org/10.1016/B978-012109890-2/50031-7

[34] Denissen, J.J.A., Penke, L. and Wood, D. (2013) Lifespan Personality Development Is Driven by Momentary Self-Regulation Dynamics. Unpublished Manuscript, Tilburg University, Tilburg.

[35] Madhuri (2012) Comparison of Personality of Alcoholics \& Drug Addicts versus Non-Alcoholics \& Non-Drug Addicts. International Journal of Science and Research (IJSR), 1, 27-35.

[36] Hurlburt, G., Gade, E. and Fuqui, D. (1982) Intercorrelational Structure of the Personality Questionnaire with an Alcoholic Population. Psychological Reports, 51, 515-520. http://dx.doi.org/10.2466/pr0.1982.51.2.515

[37] Spielberger, C.D. and Jacobs, G.A. (1982) Personality and Smoking Behavior. Journal of Personality Assessment, 46, 396-403. http://dx.doi.org/10.1207/s15327752jpa4604 11

[38] Abu-Arab, M. and Hashem, E. (1995) Some Personality Correlates in a Group of Drug Addicts. Personality and Individual Differences, 19, 649-653. http://dx.doi.org/10.1016/0191-8869(95)00115-M 